AIAA-2003-3299

\title{
Mean Flow Boundary Conditions for Computational Aeroacoustics
}

\author{
R. Hixon* \\ Mechanical, Industrial, and Manufacturing Engineering Department \\ University of Toledo \\ Toledo, $\mathrm{OH} 43606$ \\ email: dhixon@eng.utoledo.edu \\ M. Nallasamy** \\ QSS Group, Inc. \\ Mail Stop 500-QSS \\ NASA Glenn Research Center \\ Cleveland, $\mathrm{OH} 44135$ \\ S. Sawyer*** \\ Mechanical Engineering Department \\ University of Akron \\ Akron, $\mathrm{OH} 44325$ \\ R. Dyson**** \\ Mail Stop 54-3 \\ NASA Glenn Research Center \\ Brook Park, OH 44135
}

\begin{abstract}
$\underline{\text { Abstract }}$
In this work, a new type of boundary condition for time-accurate Computational Aeroacoustics solvers is described. This boundary condition is designed to complement the existing nonreflective boundary conditions while ensuring that the correct mean flow conditions are maintained throughout the flow calculation. Results are shown for a loaded 2D cascade, started with various initial conditions.
\end{abstract}

\section{Introduction}

The field of Computational Aeroacoustics

* Assistant Professor, Member AIAA

** Senior Member AIAA

***Assistant Professor, Member AIAA

**** Member AIAA

C2003 by the American Institute of Aeronautics and Astronautics, Inc. All rights reserved.
(CAA) is concerned with the time-accurate calculation of unsteady flow fields. Theflow problems of interest can be divided into two types: initial-value, and long-time unsteady problems. In an initialvalue problem, the initial flow solution is known exactly, and the computation is focused on obtaining a time-accurate unsteady solutio throughout the calculation. In a long-time unsteady problem, the initial flow field is not

This is a preprint or reprint of a paper intended for presentation at a conference. Because changes may be made before formal publication, this is made available with the understanding that it will not be cited or reproduced without the permission of the author. 
correctly specified, and the solution must evolve over time until it converges to the long-time unsteady flow.

It is relatively rare to solve an initial-value flow problem, mainly because the initial solution must

In the first phase, an initial flow solution is specified; since this is generally not the correct long-time solution, the flow evolves through a transient phase before converging to a long-time unsteady solution. During the transient phase, the goal is to converge the flow to the long-time unsteady solution rapidly; thus the transient computation need not be highly accurate in time.

Once the flow has converged to the long-time unsteady solution, the second phase of the computation begins. Here, the desired unsteady flow data is gathered; thus, a highly time-accurate calculation is desired. The data gathered in this phase is the desired output for the flow simulation.

The boundary conditions used for each phase of the calculation should be chosen to achieve the goals of each phase. Currently, nonreflective or damping boundary conditions are used for most CAA calculations. ${ }^{1}$ These conditions are designed to allow outgoing disturbances to exit the computational domain without generating either real or spurious incoming disturbances. These conditions are ideal for either an initial-value computation or the data-gathering phase of a longtime unsteady computation, when the flow solution correctly represents the desired mean flow.

However, these nonreflective boundary conditions have no mechanism for maintaining a desired mean flow when implemented in a nonlinear flow solver. In the initial transient phase, large disturbances may propagate through the boundary and exit the domain. These disturbances affect the flow solution at the boundary, and the correct method to 'reset' the flow at the boundary is to impose an incoming disturbance originating outside of the computational domain.

Notice that in this work, the mean flow boundary condition (MFBC) does not act on the be exactly specified. On the other hand, many realistic flow problems can be classified as longtime unsteady. Unlike an initial-value problem, where the entire calculation must be highly accurate, the long-time unsteady flow calculation can be divided into two distinct phases.

instantaneous flow solution at the boundary; instead, it acts on the time-averaged mean flow at the boundary. In this way, the instantaneous outgoing waves that do not affect the mean flow will not be reflected.

\section{Governing Equations and Numerical Method}

In this work, the Euler equations are solved. The 2D nonlinear Euler equations may be written in Cartesian form as:

$$
Q_{t}+E_{x}+F_{y}=0
$$

The NASA Glenn Research Center BASS code was used to solve this equation. ${ }^{2-5}$ The BASS code uses optimized explicit time marching combined with high-accuracy finite-differences to accurately compute the unsteady flow. The code is parallel, and uses a block-structured curvilinear grid to represent the physical flow domain. A constant-coefficient $10^{\text {th }}$ order artificial dissipation model $^{6}$ is used to remove unresolved highfrequency modes from the computed solution.

The BASS code solves the Euler equations using the nonconservative chain-rule formulation; previous experience has indicated that the formal lack of conservation is offset by the increased accuracy of the transformed equations. ${ }^{7,8}$ The chain-rule form of the Euler equations are:

$$
\begin{gathered}
Q_{i}+\xi_{i} Q_{\xi}+\eta_{i} Q_{\eta} \\
+\xi_{x} E_{\xi}+\eta_{x} E_{\eta} \\
+\xi_{y} F_{\xi}+\eta_{y} F_{\eta}=0
\end{gathered}
$$

For this work, the optimized low-storage RK56 scheme of Stanescu and Habashi ${ }^{9}$ was combined with the prefactored sixth-order compact differencing scheme of Hixon ${ }^{10}$. 


\section{Boundary Condition Formulation}

At the inflow and outflow boundaries of the computational domain, boundary conditions must be specified. These boundary conditions should, at a minimum, maintain the desired mean flow, be nonreflective for transient waves that do not affect the mean flow, and introduce any user-specified incoming disturbances. To accomplish these goals, the time derivative of the flow at the boundary is decomposed into three parts:

$$
\left(Q_{t}\right)_{\text {boundary }}=\left[\begin{array}{c}
\left(\bar{Q}_{t}\right)_{M F B C} \\
+\left(Q_{t}\right)_{\text {incoming }} \\
+\left(Q_{t}\right)_{\text {nonreflective }}
\end{array}\right]
$$

Thus, there are three components to the boundary conditions. The first component (MFBC) maintains the desired mean flow. The second component (incoming) introduces any user-specified disturbances. The third component (nonreflective) is nonreflective to transient waves.

The nonreflective boundary condition used in this work was the Giles inflow and outflow conditions. ${ }^{12}$ This boundary condition has been previously tested on a benchmark flat plate cascade with acceptable results. ${ }^{7}$

The incoming gust specification boundary condition is also implemented in the BASS code, though it was not used for this test. This condition is simply designed to remove the incoming gust disturbances from the Giles condition calculation, and then add the time derivative of the gust components in at the inflow boundary. This condition has been previously validated. ${ }^{7,8}$
The mean flow boundary condition is formulated in this way. First, the mean flow must be defined. The mean flow is simply the flow variables integrated over a given length of time, $\mathrm{T}$ :

$$
\bar{Q} T=\int_{t-T}^{t} Q d t
$$

Thus, the mean flow integration can be performed using the same time integration method used in the code.

Next, the desired mean flow conditions must be specified. For this problem, the desired conditions are specified in terms of the inflow stagnation temperature and pressure, the inflow angle, and the outflow static pressure.

Finally, the mean flow boundary condition must be defined. To accomplish this, the 1-D characteristics of Thompson ${ }^{13,14}$ are used, modified for an arbitrary rotation of the grid boundary:

$$
\begin{gathered}
\Delta A_{1}=(\Delta \bar{p})-\bar{c}^{2}(\Delta \bar{\rho}) \\
\Delta A_{2}=(\Delta \bar{V} \cdot m) \\
\Delta A_{3}=(\Delta \bar{p})+\bar{\rho} \bar{c}(\Delta \bar{V} \cdot n) \\
\Delta A_{4}=(\Delta \bar{p})-\bar{\rho} \bar{c}(\Delta \bar{V} \cdot n)
\end{gathered}
$$

Here, the " $n$ " vector is normal to the grid boundary, while the " $\mathrm{m}$ " vector is tangent to the grid boundary.

At each boundary point, a ' target'mean flow is defined; the object of the mean flow boundary condition is to obtain the ' target' mean flow. At the inflow boundary, the current mean flow error can be defined as: 


$$
\begin{gathered}
\Delta \bar{\alpha}=\bar{\alpha}_{\text {target }}-\bar{\alpha}_{\text {current }} \\
\Delta \bar{p}_{0}=\left(\bar{p}_{0}\right)_{\text {target }}-\left(\bar{p}_{0}\right)_{\text {current }} \\
\Delta \bar{T}_{0}=\left(\bar{T}_{0}\right)_{\text {target }}-\left(\bar{T}_{0}\right)_{\text {current }}
\end{gathered}
$$

Given that the error in the current mean flow is known, then the incoming characteristics that will correct this error can be calculated. At a subsonic inflow boundary, this results in a $4 \times 4$ matrix:

$$
[B]\left(\begin{array}{c}
\Delta A_{1} \\
\Delta A_{2} \\
\Delta A_{3} \\
\Delta A_{4}
\end{array}\right)=\left(\begin{array}{c}
\Delta \bar{p}_{0} \\
\Delta \bar{T}_{0} \\
\Delta \bar{\alpha} \\
0
\end{array}\right)
$$

where

$$
[B]=\left[\begin{array}{cccc}
\frac{\delta \bar{p}_{0}}{\delta\left(A_{1}\right)} & \frac{\delta \bar{p}_{0}}{\delta\left(A_{2}\right)} & \frac{\delta \bar{p}_{0}}{\delta\left(A_{3}\right)} & \frac{\delta \bar{p}_{0}}{\delta\left(A_{4}\right)} \\
\frac{\delta \bar{T}_{0}}{\delta\left(A_{1}\right)} & \frac{\delta \bar{T}_{0}}{\delta\left(A_{2}\right)} & \frac{\delta \bar{T}_{0}}{\delta\left(A_{3}\right)} & \frac{\delta \bar{T}_{0}}{\delta\left(A_{4}\right)} \\
\frac{\delta \bar{\alpha}}{\delta\left(A_{1}\right)} & \frac{\delta \bar{\alpha}}{\delta\left(A_{2}\right)} & \frac{\delta \bar{\alpha}}{\delta\left(A_{3}\right)} & \frac{\delta \bar{\alpha}_{4}}{\delta\left(A_{4}\right)} \\
0 & 0 & 1
\end{array}\right]
$$

Since the fourth characteristic is outgoing, it cannot be modified.

For the outflow boundary, The development is similar. Here, the matrix equation becomes:

$$
[C]\left(\begin{array}{c}
\Delta A_{1} \\
\Delta A_{2} \\
\Delta A_{3} \\
\Delta A_{4}
\end{array}\right)=\left(\begin{array}{c}
0 \\
0 \\
0 \\
\Delta \bar{p}
\end{array}\right)
$$

where

$$
[C]=\left[\begin{array}{cccc}
1 & 0 & 0 & 0 \\
0 & 1 & 0 & 0 \\
0 & 0 & 1 & 0 \\
\frac{\delta \bar{p}}{\delta\left(A_{1}\right)} \frac{\delta \bar{p}}{\delta\left(A_{2}\right)} \frac{\delta \bar{p}}{\delta\left(A_{3}\right)} \frac{\delta \bar{p}}{\delta\left(A_{4}\right)}
\end{array}\right]
$$

In constrast to the inflow boundary, now the first three characteristics are outgoing, and cannot be modified.

Once the modifications to the characteristics are known, the necessary modifications to the flow variables may be calculated as:

$$
\Delta Q=\left[\begin{array}{l}
\left(\frac{\delta Q}{\delta A_{1}}\right) \Delta A_{1} \\
+\left(\frac{\delta Q}{\delta A_{2}}\right) \Delta A_{2} \\
+\left(\frac{\delta Q}{\delta A_{3}}\right) \Delta A_{3} \\
+\left(\frac{\delta Q}{\delta A_{4}}\right) \Delta A_{4}
\end{array}\right]
$$

Finally, the mean flow boundary condition is written as: 


$$
\left(\bar{Q}_{t}\right)=\sigma(\Delta Q)
$$

The value of $\sigma$ used in this work was 1.0. Initially, much higher values of $\sigma$ were used, in an attempt to converge the mean flow solution more quickly. However, it was found that instabilities occurred during the first phase of the calculation, when very large transients were exiting the boundaries. In future work, a variable $\sigma$ will be tried, where the value of $\sigma$ is initially low to allow the large transients to leave the domain and then is increased in order to more quickly converge the mean flow solution.

\section{Test Problem and Flow Initialization}

The test problem chosen represents the type of flow problem that the BASS code is designed to solve. In this problem, the mean flow about a loaded $2 \mathrm{D}$ cascade is calculated. The cascade geometry is given in Ref. (11). The mean flow conditions at the inflow are:

$$
\begin{aligned}
& \bar{p}_{0}=1.0 \\
& \bar{T}_{0}=1.0 \\
& \bar{\alpha}=36^{\circ}
\end{aligned}
$$

At the outflow,

$$
\bar{p}=0.92
$$

In the full problem, which is a benchmark problem for the $4^{\text {th }}$ Computational Aeroacoustic Workshop on Benchmark Problems, three simple-harmonic gusts are introduced at the inflow boundary. For this work, the gusts are neglected; in future work, the gusts will be included in the calculation.
In order to run the a time-marching flow code, an initial flow condition must be specified. For this work, seven initial conditions were used, in which the flow is uniform throughout the computational domain. These seven initial conditions are referred to as Tests 1-7, as defined in Table 1. To validate the mean flow boundary conditions further, an additional four tests were run in which the initial condition of Test 1 was used and the desired mean flow conditions were varied. These cases are referred to as Tests 8-11, as defined in Table 1:

Table 1: Flow Test Cases

\begin{tabular}{|c|c|c|c|c|}
\hline Test & $\bar{p}_{0}$ & $\bar{T}_{0}$ & $\bar{\alpha}$ & $\bar{p}$ \\
\hline 1 & 1 & 1 & 36 & 0.92 \\
\hline 2 & 1 & 1 & 30 & 0.92 \\
\hline 3 & 1 & 1 & 35 & 0.92 \\
\hline 4 & 1 & 1 & 40 & 0.92 \\
\hline 5 & 1 & 1 & 36 & 0.85 \\
\hline 6 & 0.95 & 1 & 36 & 0.92 \\
\hline 7 & 1 & 0.95 & 36 & 0.92 \\
\hline 8 & 1 & 1 & 32 & 0.92 \\
\hline 9 & 0.95 & 1 & 36 & 0.92 \\
\hline 10 & 1 & 0.95 & 36 & 0.92 \\
\hline 11 & 1 & 1 & 36 & 0.85 \\
\hline
\end{tabular}

\section{$\underline{\text { Results }}$}

The first seven test cases given in Table 1 were run to test the ability of the MFBC to obtain the correct mean flow while starting from different initial conditions. For comparison, these cases were also run without the mean flow boundary condition.

Figures 1-7 show the convergence histories of each mean flow variable at one point on 
the inflow and outflow boundaries for each test case. Each figure compares the mean flow obtained both with and without the MFBC enabled. It is clearly seen that the mean flow is not remaining constant through the run, and the effect of the MFBC is to bring the computed flow to the correct mean values. Also, the convergence to the mean flow as the run progresses is shown. This is very important, as it indicates that the MFBC is only active during the initial transient portion of the code run.

Another important point is that the MFBC was used in conjunction with the Giles nonreflective boundary condition; the results show the utility of using both a mean flow and an instantaneous nonreflective boundary condition.

The final four tests (Tests 8-11) were designed to investigate the ability of the MFBC to obtain different desired mean flows while starting from the same initial condition. Figure 8 illustrates the time history of the four runs, and the final mean flows are those given in Table 1.

\section{Conclusions and Future Directions}

In this work, a mean flow boundary condition for time-marching unsteady calculations was developed. The initial tests showed the effectiveness of this boundary condition; the mean flow converged to the correct conditions regardless of the initial flow condition. This will be a useful addition to any nonlinear unsteady code using nonreflective boundary conditions, since these nonreflective boundary conditions do not automatically maintain the desired mean flow.

In future, the user-input unsteady gust will be added to the flow, and a full unsteady nonlinear calculation will be performed. This will provide the final test for the mean flow boundary conditions.

\section{Acknowledgments}

This work was funded by the NASA Glenn Research Center under the Quiet Aircraft Technology program. The authors would like to thank Dr. Edmane Envia and Dr. Christopher
Miller of the NASA Glenn Research Center for their contributions to this effort.

\section{$\underline{\text { References }}$}

1) Hixon, R. ' Radiation and Wall Boundary Conditions for Computational Aeroacoustics: A Review', International Journal of Computational Fluid Dynamics, 2003.

2) Hixon, R, Nallasamy, M., and Sawyer, S., ' Parallelization Strategy for an Explicit Computational Aeroacoustics Code' , AIAA Paper 2002-2583, July 2002.

3) Hixon, R., Nallasamy, M., and Sawyer, S., ' Effectof Grid Singularities on the Solution Accuracy of a CAA Code' AIAA Paper 20030879, Jan. 2003.

4) Nallasamy, M., Hixon, R., Sawyer, S., Dyson, R., and Koch, L., ' AParallel Simulation of Rotor Wake - Stator Interaction Noise' AIAA Paper 2003-3134, May 2003.

5) Sawyer, S., Nallasamy, M., Hixon, R., Dyson, R., and Koch, D., ' ComputationaAeroacoustic Prediction of Discrete-Frequency Noise Generated by a Rotor-Stator Interaction' AIAA Paper 2003-3268, May 2003.

6) Kennedy, C. A. and Carpenter, M. H., ' Several New Numerical Methods for Compressible Shear-Layer Simulations' Applied Numerical Mathematics, Vol. 14, 1994, pp. 397-433.

7) Hixon, R., Shih, S.-H., and Mankbadi, R. R., 'Evaluation of Boundary Conditions for the Gust- Cascade Problem', Journal of Propulsion and Power, Vol. 16, No. 1, 2000, pp. 72-78.

8) Hixon, R., Mankbadi, R. R., and Scott, J. R., - Validation of a High-Order Prefactored Compact Code on Nonlinear Flows with Complex Geometries' AIAA Paper 2001-1103, Jan. 2001.

9) Stanescu, D. and Habashi, W. G., ' 2N-Storage Low Dissipation and Dispersion Runge- Kutta Schemes for Computational Acoustics', Journal of Computational Physics, Vol. 143, No. 2, 1998, p. 674-681.

10)Hixon, R., ' Prefactore Small-Stencil Compact Schemes' Journal of Computational Physics, Vol. 165, 2000, p. 522-541.

11)www.math.fsu.edu/caa4

12)Giles, M. B., ' Nonreflecting Boundary 
Conditions for Euler Equation Calculations' ,AIAA Journal, Vol. 28, No. 12, 1990, pp. 2050-2058.

13)Thompson, K. W., ' TimeDependent Boundary Conditions for Hyperbolic Systems' , Journal of Computational Physics, Vol. 68, No. 1, 1987, pp. 1-24.

14) Thompson, K. W., ' TimeDependent Boundary Conditions for Hyperbolic Systems II' Journal of Computational Physics, Vol. 89, 1990, pp. 439-461.

\section{Figures}

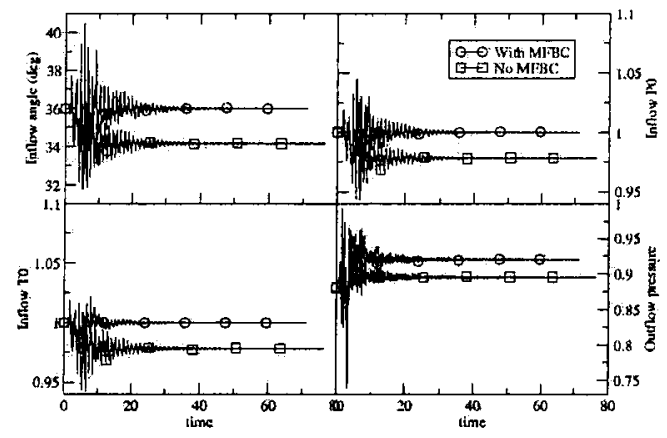

Figure 1: Mean Flow Test Case 1

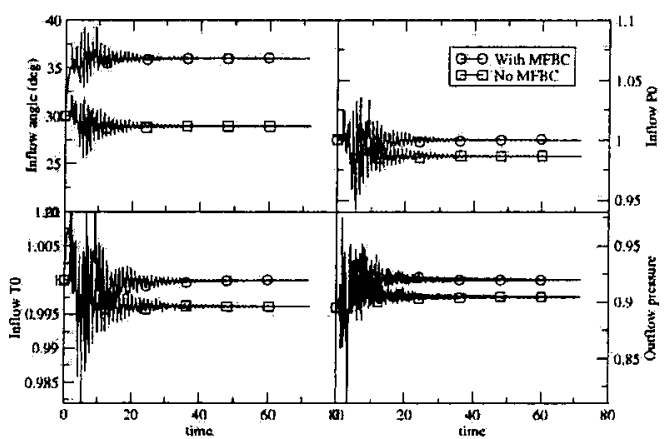

Figure 2: Mean Flow Test Case 2

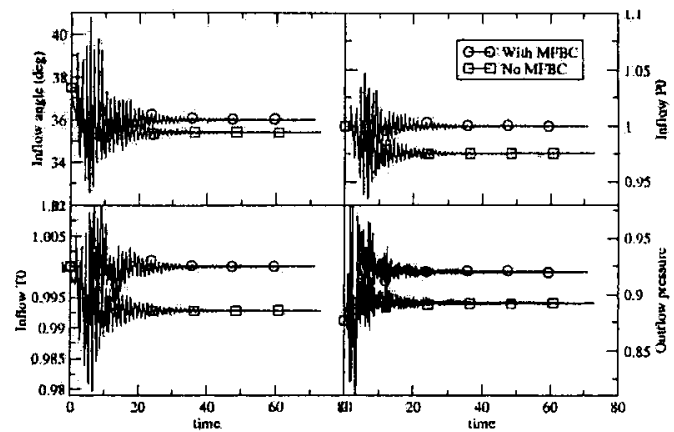

Figure 3: Mean Flow Test Case 3 


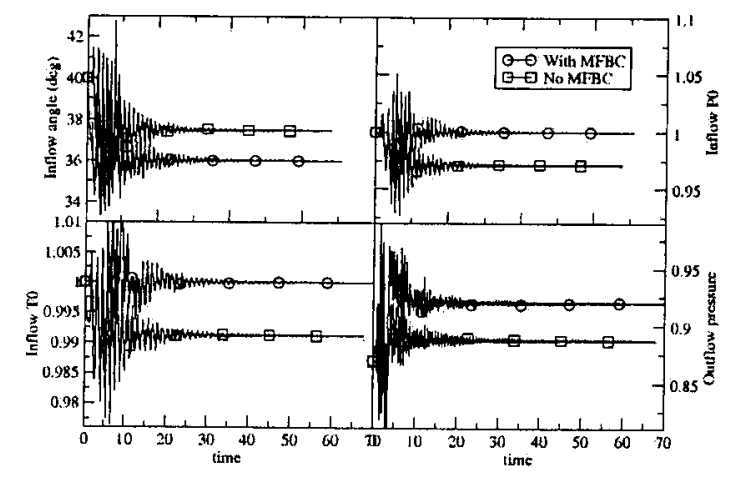

Figure 4: Mean Flow Test Case 4

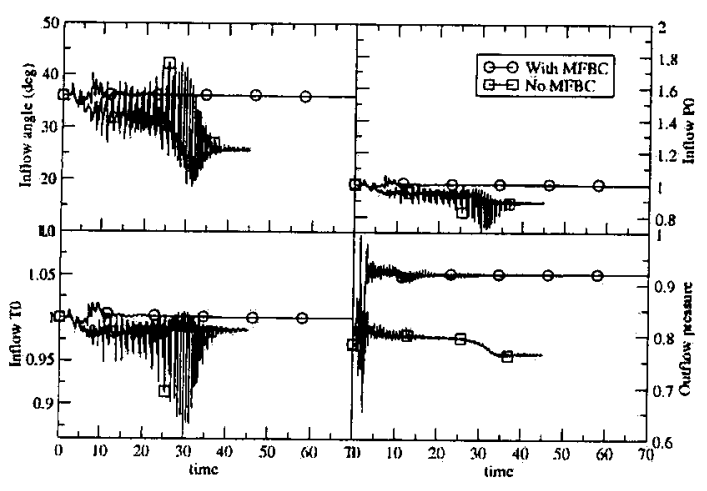

Figure 5: Mean Flow Test Case 5

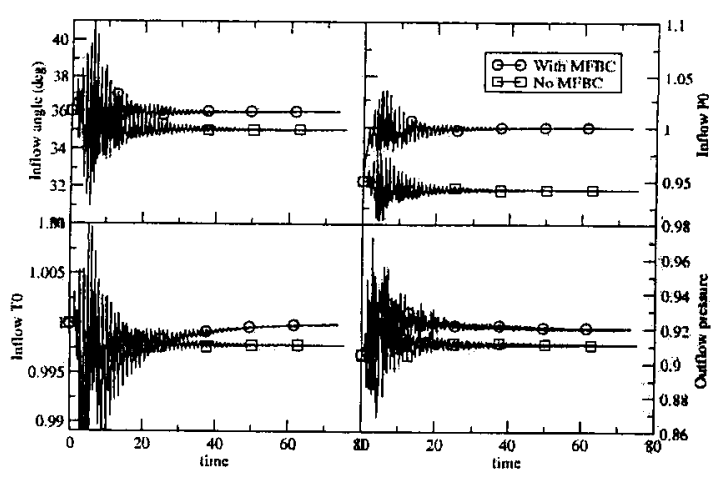

Figure 6: Mean Flow Test Case 6

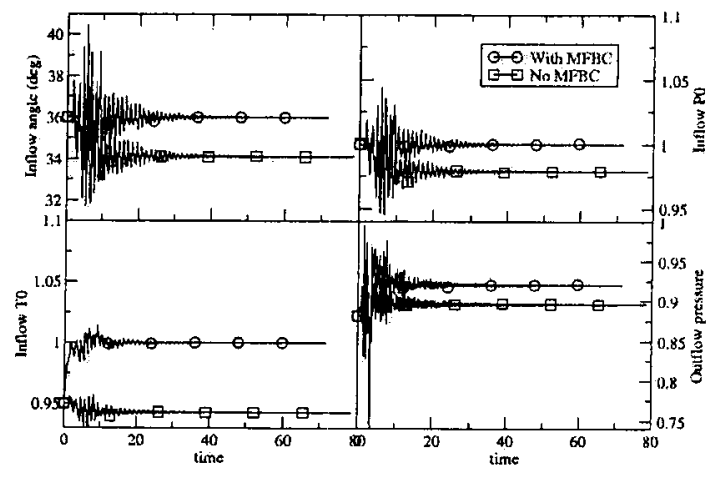

Figure 7: Mean Flow Test Case 7

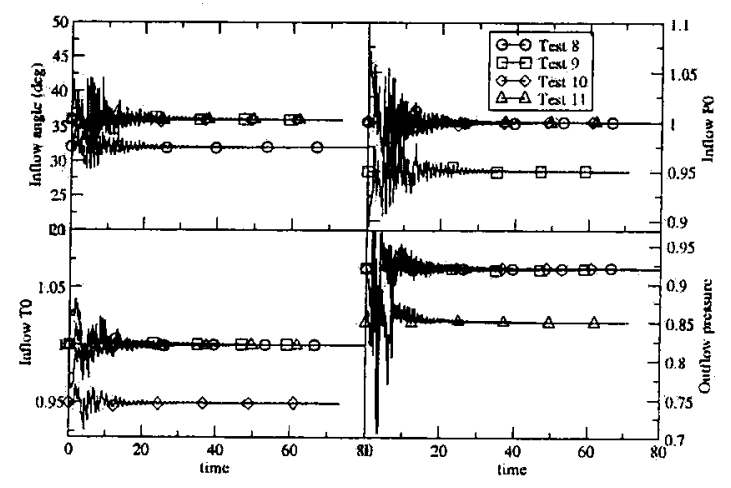

Figure 8: Mean Flow Test Cases 8-11 